\title{
Explaining the link between adiposity and colorectal cancer risk in men and postmenopausal women in the UK Biobank: a sequential causal mediation analysis
}

S Ghazaleh Dashti ${ }^{1,2}$, Vivian Viallon ${ }^{2}$, Julie A Simpson ${ }^{1}$, Amalia Karahalios ${ }^{1,3}$, Margarita MorenoBetancur $^{4,5}$, Dallas R English ${ }^{1,6}$, Marc J Gunter ${ }^{2}$, Neil Murphy ${ }^{2}$

1 Centre for Epidemiology and Biostatistics, Melbourne School of Population and Global Health, The University of Melbourne, Melbourne, Victoria, Australia

2 Section of Nutrition and Metabolism, International Agency for Research on Cancer (IARC), Lyon, France

3 School of Public Health and Preventive Medicine, Monash University, Melbourne, Victoria, Australia

4 Clinical Epidemiology and Biostatistics Unit, Murdoch Children's Research Institute, Melbourne,

Australia

5 Department of Pediatrics, The University of Melbourne, Melbourne, Victoria, Australia

6 Cancer Epidemiology and Intelligence Division, Cancer Council Victoria, Melbourne, Victoria, Australia

\section{Corresponding author:}

S Ghazaleh Dashti

Centre for Epidemiology and Biostatistics

Melbourne School of Population and Global Health

Level 3, 207 Bouverie Street

The University of Melbourne VIC 3010 Australia

Email: seyedeh.dashti@unimelb.edu.au

\section{Short title: Explaining adiposity and colorectal cancer link}

Key words: adiposity, colorectal cancer, causal mediation analysis, C-reactive protein, inflammation, hemoglobin-A1c, insulin resistance, sex-steroid hormones, sex hormone-binding globulin, testosterone

Article category: Cancer Epidemiology

\section{Abbreviations used:}

CRC: colorectal cancer; CRP: C-reactive protein; HbA1c: hemoglobin-A1c; SHBG: sex hormone binding globulin; NDE: natural direct effect; NIE: natural indirect effect; RR: risk ratio; CI: confidence interval; ICD: International Classification of Disease; IQC: internal quality control; CV: coefficient of variation; WHO: World Health Organization; GMR: geometric mean ratio; OR: odds ratio; TE: total effect; IPW: inverse probability weights; MI: multiple imputation; HPFS: Health Professionals Follow-up Study; sOB-R: soluble leptin receptor; HR: hazard ratio; TyG: triglyceride-glucose index

\section{Novelty and Impact}

Mechanisms underlying adiposity-colorectal cancer (CRC) association are not well understood. Here, we used UK Biobank data and performed sequential mediation analysis to quantify mediating effects of Creactive protein, hemoglobin-A1c, sex-hormone-binding globulin and testosterone in this association in men and postmenopausal women. Our results suggest pathways marked by these obesity-related factors may not

This is the author manuscript accepted for publication and has undergone full peer review but has not been through the copyediting, typesetting, pagination and proofreading process, which may lead to differences between this version and the Version of Record. Please cite this article as doi: $10.1002 / \mathrm{ijc} .32980$ 
explain a large proportion of this association and pathways other than those captured here may also be important for understanding the adiposity-CRC link.

This article is protected by copyright. All rights reserved. 


\section{Abstract}

Mechanisms underlying adiposity-colorectal cancer (CRC) association are incompletely understood. Using UK Biobank data, we investigated the role of C-reactive protein (CRP), hemoglobin-A1c (HbA1c), and (jointly) sex hormone-binding globulin (SHBG) and testosterone, in explaining this association. Total effect of obesity vs normal-weight (based on waist circumference, body mass index, waist-hip ratio) on CRC risk was decomposed into natural direct (NDE) and indirect (NIE) effects using sequential mediation analysis. After a median follow-up of 7.1 years, 2070 incident CRC cases (men=1280; postmenopausal women=790) were recorded. For men, the adjusted risk ratio (RR) for waist circumference ( $\geq 102$ vs $\leq 94 \mathrm{~cm}$ ) was 1.37 (95\% confidence interval (CI),1.19-1.58). The RRs ${ }^{\mathrm{NIE}}$ were 1.08 (95\%CI,1.01-1.16) through all biomarkers, 1.06 (95\%CI,1.01-1.11) through pathways influenced by CRP, 0.99 (95\%CI,0.97-1.01) through HbA1c beyond [the potential influence of] CRP, and 1.03 (95\%CI,0.99-1.08) through SHBG and testosterone combined beyond CRP and HbA1c. The RR ${ }^{\mathrm{NDE}}$ was 1.26 (95\%CI,1.09-1.47). For women, the RR for waist circumference ( $\geq 88$ vs $\leq 80 \mathrm{~cm}$ ) was 1.27 (95\%CI,1.07-1.50). The RRs ${ }^{\mathrm{NIE}}$ were 1.08 (95\%CI,0.94-1.22) through all biomarkers, 1.08 (95\%CI,0.99-1.17) through CRP, 1.00 (95\%CI,0.98-1.02) through HbA1c beyond CRP, and 1.00 (95\%CI;0.92-1.09) through SHBG and testosterone combined beyond CRP and HbA1c. The RR ${ }^{\mathrm{NDE}}$ was 1.18 (95\%CI,0.96-1.45). For men and women, pathways influenced by CRP explained a small proportion of the adiposity-CRC association. Testosterone and SHBG also explained a small proportion of this association in men. These results suggest that pathways marked by these obesityrelated factors may not explain a large proportion of the adiposity-CRC association. 


\section{Introduction}

Globally, colorectal cancer (CRC) is the third most commonly diagnosed cancer among men and women ${ }^{1}$. There is a wide disparity in CRC incidence across the world regions. High-income countries have three times higher incidence rate compared with low- and middle-income countries ${ }^{1}$, while the latter, especially countries undergoing significant developments, have faced an increased burden of CRC more recently ${ }^{2}$. These patterns highlight the role that some of the consequences of a 'Westernized' lifestyle may have in developing CRC ${ }^{1}$.

Excess body fatness, a hallmark of 'Westernized' lifestyle, is an established risk factor for CRC, as well as for 12 other cancers ${ }^{3}$. Based on a recent meta-analysis, the relative risk (RR) of CRC for the highest vs lowest category of waist circumference (a measure of central adiposity) was 1.48 (95\% confidence interval (CI), 1.30-1.67) for men and 1.44 (95\%CI, 1.30-1.60) for women ${ }^{4}$. Similar associations were reported for BMI (obese vs normal), with a more evident difference in the strength of association for men (RR 1.47; 95\%CI, 1.36-1.58) compared with women (RR 1.15; 95\%CI, 1.08-1.23) ${ }^{4}$.

Overweight and obesity already make a substantial contribution to the burden of CRC. In 2012, 13\% (90\% confidence interval (CI), 11\%-14\%) of colon and 6\% (90\%CI, 5\%-7\%) of rectal cancer cases diagnosed in men and 8\% (90\%CI, 7\%-8\%) of colon and 4\% (90\%CI, 3\%-4\%) of rectal cancer cases diagnosed in women were attributable to overweight and obesity $\left(\mathrm{BMI} \geq 25 \mathrm{~kg} / \mathrm{m}^{2}\right)^{5}$. Globally, the prevalence of adults living with obesity (BMI $>30 \mathrm{~kg} / \mathrm{m}^{2}$ ) has increased from $\sim 3 \%$ in 1975 to $11 \%$ in 2014 in men and from $\sim 6 \%$ in 1975 to $15 \%$ in 2014 in women ${ }^{6}$. If these trends continue, by 2025, 18\% of men and $21 \%$ of women will have obesity ${ }^{7}$. It can, therefore, be expected for obesity to make considerably larger contributions to CRC burden in the future.

This article is protected by copyright. All rights reserved. 
Adiposity-induced chronic inflammation, dysregulation of insulin signaling and glucose homeostasis, and sex-steroid hormones production are three interconnected pathways hypothesized to mediate the adiposityCRC association ${ }^{8}$. Inflammation might exert its influence on CRC risk directly (through its angiogenic, mitogenic, and antiapoptotic effects) ${ }^{8}$ and through contributions to the development of insulin resistance and dysregulated insulin signaling ${ }^{9}$ and sex-steroid hormones production pathways ${ }^{10,11}$. Insulin resistance may increase cancer risk through hyperglycemia and exposure to elevated levels of insulin which has mitogenic and antiapoptotic effects ${ }^{8}$ and its influences on insulin-like growth factor- ${ }^{12}{ }^{13}$, and sex-steroid hormones levels ${ }^{10,11,14}$. The potential role of sex-steroid hormones in colorectal carcinogenesis is not wellunderstood, but the higher incidence of CRC in men, a stronger adiposity-CRC association in men ${ }^{8}$, differences in the associations between adiposity and sex-steroid hormone levels in men and women (inverse association with testosterone levels in men, positive association in women) ${ }^{15}$, and the inverse association between hormone therapy and CRC in women ${ }^{16}$ are consistent with a role of sex-steroid hormones in the etiology of $\mathrm{CRC}^{8}$. Overall, however, the evidence for associations between sex-steroid hormones and CRC risk in men and women is limited and inconclusive ${ }^{8}$.

We used data from the UK Biobank to investigate the extent to which biomarkers of these three pathways mediate the effect of adiposity (measured by waist circumference, BMI, and waist-hip ratio) on CRC risk in men and postmenopausal women. A sequential causal mediation analysis approach was used to quantify the mediating effects through the three pathways without assuming they acted independently ${ }^{17}$. The assessed biomarkers were high sensitivity C-reactive protein (CRP, a non-specific biomarker of systemic inflammation), glycated hemoglobin (HbA1c, a biomarker of glycemic control and a correlate of insulin resistance ${ }^{18}$ ), and sex-hormone-binding globulin (SHBG) and testosterone (treated jointly to capture some aspects of altered sex-steroid hormone levels in the obese state).

\section{Methods}


The UK Biobank is a prospective cohort of 229,134 men and 273,402 women recruited between 2006 and 2010 following an invitation letter sent to about 9.2 million individuals ( $~ 5.5 \%$ response rate $)^{19}$. Invitees were registered with the UK National Health Service, were mostly 40-69 years old, and lived within approximately 25 miles of one of 22 assessment centers in England, Wales, and Scotland. Participants gave informed consent, and the North West Multi-Centre Research Ethics committee, the National Information Governance Board for Health and Social Care in England and Wales, and the Community Health Index Advisory Group in Scotland approved the study. In 2004, the Ethics and Governance Council was established to ensure adherence to the Ethics and Governance Framework (http://www.ukbiobank.ac.uk/ethics/) of the study. This research was conducted using the UK Biobank Resource under application number 25897.

The current study used data from all participants, with the outcome defined as CRC diagnosis between one year after baseline (i.e. recruitment) and end of follow-up (see Follow-up and outcome assessment).

\section{Data collection}

At baseline, information on demographics, early life and lifestyle exposures, health history and medication use were collected using a self-administered touchscreen questionnaire available at http://www.ukbiobank.ac.uk/key-documents/. Weight, height, waist and hip circumference were measured following prespecified protocols ${ }^{20}$, and blood samples were collected, centrifuged, and stored at $-80^{\circ} \mathrm{C}$ or liquid nitrogen within $\sim 24$ hours after venipuncture ${ }^{21}$.

\section{Follow-up and outcome assessment}

Incident cancer cases and cancer cases recorded first in death certificates were identified through linkage to national cancer and death registries. Complete follow-up was available through 31 March 2016 for England 
and Wales and 31 October 2015 for Scotland. The 10th Revision of the International Classification of Disease (ICD-10) was used to define CRC cases (C18, C19, and C20 for malignant neoplasms of colon, rectosigmoid junction, and rectum respectively).

\section{Selection of participants eligible for the study}

Participants were ineligible for this study if they had withdrawn consent; had cancer diagnosed before baseline; had less than one-year follow-up; were diagnosed with CRC within the first year after baseline; were diagnosed with cancer of the appendix (ICD-10 C18.1; $<1 \%$ ); died (<2\%) or were lost to follow-up

$(<0.5 \%)$ before the end of follow-up; did not have body size measurements collected or had BMI $<18.5$ $\mathrm{kg} / \mathrm{m}^{2}$ at baseline; had unknown diabetes status, had diabetes or reported taking diabetes medication at baseline (Figure 1a-1b). The last exclusion criterium was imposed because diabetes and diabetes medication use may change the link between adiposity and insulin signaling ${ }^{22}$. Additionally, women who, at baseline, were premenopausal or $\leq 55$ years old with unknown menopause status, who reported taking hormone therapy, or hormonal contraceptives were ineligible (Figure 1b). We did not include premenopausal women in this analysis because the mechanistic pathways, especially the sex-steroid hormones pathway, might be different in pre- and postmenopausal women. Instead of multiply imputing missing confounder data (the approach used to handle missing biomarker data), we also excluded participants with missing data for any of the selected confounders (see Confounder selection) because they were a relatively small proportion $(\leq 10 \%)$ of all the participants.

\section{Biomarkers and laboratory methods}

Descriptions of laboratory methods and protocols for measuring the biomarkers have been published ${ }^{23,}{ }^{24}$. We did not include estradiol in our analyses because most participants had poor quality data ${ }^{24}$. 
Measurements were performed in blood samples (red blood cells for HbA1c, serum for all other biomarkers) collected at baseline (2006-2010) as part of the UK Biobank Biomarker Project. Immuno-turbidimetric metric (Beckman Coulter AU5800) was used to measure CRP (with average within-laboratory coefficient of variation (CV) of $2.3 \%$ for low and $1.7 \%$ for high internal quality control (IQC) samples); high-performance liquid chromatography (Bio-Rad Variant II Turbo) to measure HbA1c (CV 2.1\% for low, 1.5\% for high IQC samples); and chemiluminescent immunoassay (Beckman Coulter DXI 800) to measure SHBG (CV 5.7\% for low, 5.2\% for high IQC samples), and testosterone (CV 8.3\% for low, 4.2\% for high IQC samples) ${ }^{23,24}$. An algorithm was developed to ensure each laboratory batch included a random selection of participants to reduce the likelihood of any systematic differences in assay results between participants ${ }^{24}$. When applicable, the data we received had also been corrected for aliquot-dilution effect (SHBG, testosterone) and date of the assay (HbA1c, SHBG, testosterone) ${ }^{21}$. Free testosterone was calculated from measured testosterone, SHBG, and albumin ${ }^{25}$. For a subgroup of participants, biomarker measurements were additionally performed in blood samples collected at repeat assessment visit (2012-2013). Intraclass correlation coefficients (ICC) were calculated using the two measurements to assess the reproducibility of the measures (see Statistical analysis).

\section{Statistical analysis}

Waist circumference, BMI, and waist-hip ratio were used as measures of adiposity in separate analyses. For BMI, we used the World Health Organization (WHO) recommended cut-off values to compare participants with obesity vs normal weight ( $\geq 30 v s<25 \mathrm{~kg} / \mathrm{m}^{2}$ ). For waist circumference, we also used the WHO suggested cut-off values to compare participants defined as "at substantially increased risk of metabolic complications" (referred to as with obesity hereafter) with normal ( $>102$ vs $\leq 94 \mathrm{~cm}$ for men, $>88 v s \leq 80 \mathrm{~cm}$ for women) ${ }^{26}$. For waist-hip ratio, we used sex-specific quartiles to define with obesity ( $4^{\text {th }}$ quartile $>0.98$ 
for men, $>0.86$ for women) vs normal ( $1^{\text {st }}$ quartile $\leq 0.89$ for men, $\leq 0.77$ for women) because the WHO does not have a suggested cut-off value for normal ${ }^{26}$. We treated CRC diagnosis as a binary outcome because methods for handling multiple mediators and survival outcome are not fully developed yet ${ }^{27}$.

To calculate the ICCs for biomarker measurements, linear mixed-effects modelling was used to obtain estimates of the between- and within-person variances.

Confounder Selection: The following variables were identified a priori and included as potential confounders (i.e. common causes for exposure-outcome, mediator-outcome, or exposure-mediator associations ${ }^{28}$ ): age at baseline (fitted as splines), educational attainment, Townsend deprivation index, first-degree relative history of CRC, physical activity, alcohol intake, smoking status, total red or processed meat intake, regular aspirin or ibuprofen use; additionally for women number of live births, ever use of oral contraceptive pill, and ever use of hormone therapy.

Adiposity-biomarker associations: For each biomarker, the geometric mean ratio (GMR; 95\% CI) was estimated in relation to adiposity measures (comparing with obesity vs normal) using linear regression models fitted to the log-transformed biomarker variable adjusting for potential confounders.

Adiposity-CRC \& Biomarker-CRC associations: Odds ratios (OR; 95\% CI) for the adiposity-CRC and biomarker-CRC associations were estimated using logistic regression models adjusted for confounders. For each biomarker-CRC model, we additionally included waist circumference and other biomarkers that might have confounded the association (i.e. CRP for HbaA1c-CRC model; and CRP and HbA1c for SHBG-CRC and testosterone-CRC models, selected based on the hypothesized causal sequence - see Figure 2). The linearity of the biomarker-CRC associations was investigated in models with biomarker variables fitted as restricted cubic splines (2 degrees of freedom). 
Sequential mediation analysis: Using a causal mediation analysis approach ${ }^{17}$, the total adiposity-CRC association (total effect Risk Ratio $(\mathrm{RR})^{\mathrm{TE}}$ ) was decomposed into a natural indirect effect $\left(\mathrm{RR}^{\mathrm{NIE}}\right.$; effect explained by all the included biomarkers, i.e. CRP, HbA1c, SHBG, and testosterone) and a natural direct effect ( $\mathrm{RR}^{\mathrm{NDE}}$; effect not explained by any of the included biomarkers) (see ${ }^{28}$ for a formal definition of NDE and NIE). Assuming that CRP levels might have also influenced HbA1c or biomarker of sex-steroid hormone pathway ${ }^{9,11}$, and HbA1c might have influenced biomarker of sex-steroid hormone pathway ${ }^{11,14}$, but not vice versa, we took a sequential approach to further decompose the estimated NIE into the following NIEs: i) through pathways influenced by CRP; ii) through HbA1c excluding the possible influence of CRP on HbA1c; and iii) through SHBG and testosterone combined excluding the influences of CRP or HbA1c. Figure 2 provides a schematic explanation of these effects and the sequential process used to estimate them 17.

The mediation analysis involved calculating inverse probability weights (IPWs) based on the predicted probabilities of being obese from logistic regression models for exposure conditional on confounders ${ }^{17}$. Then, under different counterfactual scenarios, potential outcomes weighted by the IPWs (to take confounding into account) were estimated based on logistic regression models for the outcome conditional on exposure, confounders, with and without the biomarkers ${ }^{17}$. Potential outcomes were expressed as risks, using the following formulae risk $=\frac{1}{(1+\exp (-\log (\text { odds })))}^{29}$. To accommodate non-linear biomarker-CRC associations, relevant biomarker variables were included as restricted cubic splines. Outcome models used to estimate each effect are presented in Figure 2.

Missing data: Missing biomarker data were handled using multiple imputation (MI) based on chained equations with 20 iterations for men and 35 for women (18\% of men and 35\% of women had missing values for at least one biomarker (Table 1)). For each adiposity measure, MI was performed with separate 
imputation models for obese and non-obese individuals ${ }^{30}$. The imputation models included all variables in the mediation analyses and the biomarker-biomarker and biomarker-CRC interaction terms (i.e. treating these interactions as ‘just another variable’ with missing values) ${ }^{30}$. Then, the obesity-biomarker interaction terms were generated from the multiply imputed datasets ${ }^{30}$.

Mediation analysis was performed for each imputed dataset and standard errors for TE, NDE, and NIEs were estimated using 1000 bootstrap samples. Rubin’s rules were then applied to calculate the final MI estimates of effects and 95\% CIs by pooling the estimated effects across the imputed datasets and deriving the standard errors from the within- and between-imputation variances ${ }^{31}$.

Additional Analyses: The following additional mediation analyses were performed: i) for the outcome models, including an interaction term between the adiposity measure and each biomarker, as well as between CRP-HbA1c, CRP-SHBG, CRP-testosterone, HbA1C-SHBG, HbA1C-testosterone, and SHBGtestosterone; ii) using calculated free testosterone as the biomarker of sex-steroid hormone pathway (instead of testosterone and SHBG combined); iii) excluding rectal cancer cases and limiting analyses to colon cancer; and iv) excluding colorectal cancer cases diagnosed within the first two years of follow-up and noncases with follow-up $\leq 2$ years.

All analyses were performed using Stata version $15^{32}$.

\section{Data availability}

UK Biobank is an open-access resource. Bonafide researchers can apply to use the UK Biobank dataset by registering and applying at http://ukbiobank.ac.uk/register-apply/.

\section{Results}


Overall, 179,936 men and 136,578 women met the eligibility criteria for this analysis (Figure 1). Men with waist circumference $>102$ vs $\leq 94 \mathrm{~cm}$ had higher median red or processed meat intake, and a higher proportion had lower physical activity levels, were former smokers, and reported regular aspirin or ibuprofen use. Similar differences were observed for women with waist circumference $>88$ vs $\leq 80$ (Table 1; see Supplementary Tables 1 and 2 for distributions of baseline characteristics by BMI and waist-hip ratio categories respectively). During a median follow-up time of 7.1 years, 1,280 and 790 CRC cases were diagnosed in men and postmenopausal women, respectively.

The ICCs (95\% CI) for the biomarkers ranged from 0.56 (95\%CI, 0.55-0.57) to 0.86 (95\%CI, 0.86-0.87) for CRP and SHBG respectively in men and 0.67 (95\%CI, 0.66-0.68) to 0.81 (95\%CI, 0.80-0.82) for CRP and SHBG respectively in women (Supplementary Table 3).

Adiposity-biomarker associations: For men with waist circumference $>102$ vs $\leq 94 \mathrm{~cm}$, an inverse association was observed for SHBG (GMR 0.79; 95\%CI, 0.79-0.79) and testosterone (GMR 0.83; 95\%CI, 0.82-0.83); a positive association for CRP (GMR 2.00; 95\%CI, 1.97-2.02); and a weak positive association for HbA1c (GMR 1.04; 95\%CI, 1.04-1.05). For women with waist circumference $>88$ vs $\leq 80 \mathrm{~cm}$, an inverse association was observed for SHBG (GMR 0.63; 95\%CI, 0.63-0.63); a positive association for CRP (GMR 2.64; 95\%CI, 2.60-2.67); and weak positive associations for testosterone (GMR 1.08; 95\%CI, 1.07-1.09) and HbA1c (GMR 1.04; 95\%CI, 1.04-1.04) (Table 2). The associations were similar for BMI and waist-hip ratio in both men and women. (See Supplementary Table 4 for complete case analysis).

Adiposity-CRC \& Biomarker-CRC associations: The adjusted OR for CRC risk for men with waist circumference $>102$ vs $\leq 94 \mathrm{~cm}$ was 1.38 (95\% CI, 1.20-1.59). Similar associations were observed for BMI and waist-hip ratio. A positive association was observed for CRP after adjusting for confounders and waist circumference (OR per doubling concentration 1.07; 95\% CI, 1.02-1.11), and there was suggestion for an 
inverse association for SHBG (OR 0.92; 95\% CI, 0.82-1.03) and testosterone (OR 0.92; 95\% CI, 0.81-1.05) after additionally adjusting for CRP, and HbA1c. There was no evidence for an association between HbA1c and CRC risk (OR additionally adjusted for CRP 0.82; 95\% CI, 0.58-1.14) (Table 3 multiple imputation analysis; Supplementary Table 5 complete case analysis). No strong evidence for departure from linearity for adiposity-CRC or biomarker-CRC associations was observed (Table3).

For women with waist circumference $>88$ vs $\leq 80 \mathrm{~cm}$, the adjusted OR for CRC was 1.28 (95\% CI, 1.081.51). A weaker association was observed for BMI ( $\geq 30$ vs $<25 \mathrm{~kg} / \mathrm{m}^{2}$, OR 1.11; 95\% CI, 0.91-1.36) and a stronger association for waist-hip ratio ( $>0.89$ vs $\leq 0.77$, OR 1.33; 95\% CI, 1.07-1.65). The point estimates and 95\% CI were suggestive of weak-to-null associations between biomarkers and CRC (adjusted OR per doubling CRP 1.03; 95\%CI, 0.98-1.09; HbA1C 1.06; 95\% CI, 0.66-1.71; SHBG 1.03, 95\%CI, 0.90-1.18; testosterone 1.00; 95\%CI, 0.88-1.13) (Table 3 multiple imputation analysis; Supplementary Table 5 complete case analysis). There was evidence for a non-linear SHBG-CRC association for women. Therefore, in the models for mediation analysis for women, SHBG was included as restricted cubic splines.

Sequential mediation analysis: For men with waist circumference $>102 v s \leq 94 \mathrm{~cm}$, the $\mathrm{RR}^{\mathrm{NIE}}$ was 1.08 (95\% CI, 1.01-1.16) through all the biomarkers, 1.06 (95\% CI, 1.01-1.11) through CRP and pathways influenced by CRP, 0.99 (95\% CI, 0.97-1.01) through HbA1c excluding the influence of CRP on HbA1c levels, and 1.03 (95\% CI, 0.99-1.08) through SHBG and testosterone combined excluding the influences of CRP and HbA1c on SHBG and testosterone levels. The RR ${ }^{\mathrm{NDE}}$ not through any of the included biomarkers was 1.26 (95\% CI, 1.09-1.47). Similar patterns of mediation were observed for BMI and waist-hip ratio (Table 4, see Supplementary Table 6 for complete case analysis).

For women, the $\mathrm{RR}^{\mathrm{NIE}}$ was 1.08 (95\% CI, 0.95-1.22) through all the biomarkers, 1.08 (95\% CI, 0.99-1.17) through pathways influenced by CRP, 1.00 (95\% CI, 0.98-1.02) through HbA1c excluding the influence of 
CRP, and 1.00 (95\% CI, 0.92-1.09) through SHBG and testosterone combined excluding the influences of CRP and HbA1c. The RR ${ }^{\mathrm{NDE}}$ not through any of the included biomarkers was 1.18 (95\% CI, 0.96-1.45). Similar mediation effects were observed for waist-hip ratio. We did not attempt to perform mediation analysis for BMI, because no total effect of BMI on CRC was observed in the multiple imputation (Table 4) or complete case analyses (Supplementary Table 6).

\section{Additional analyses}

Patterns of mediation were similar in analyses that included all possible adiposity-mediator and mediatormediator interaction terms (Supplementary Table 7), when calculated free testosterone was used as the biomarker of sex-steroid hormone pathway (Supplementary Table 8), in analyses limited to colon cancer cases (Supplementary Table 9), and in analyses limited to colorectal cancer cases diagnosed after two years of follow-up and non-cases with more than two years of follow-up (Supplementary Table 10).

\section{Potential influence of missing data}

We had missing biomarker data for 18\% of men and 35\% of women. Baseline characteristics were comparable for men with and without missing biomarker data. A slightly larger proportion of women with missing biomarker data had waist circumference $\leq 80 \mathrm{~cm}(41 \%$ vs $39 \%)$ or $\mathrm{BMI}<25 \mathrm{~kg} / \mathrm{m}^{2}$ (40\% vs $37 \%$ ) compared with women with complete biomarker data (Supplementary Table 11).

For all analyses, results from complete case analyses are presented (Supplementary Table 4 to 11). Multiple imputation and complete case analyses yielded similar results but the 95\%CIs from multiple imputation analyses were slightly narrower.

\section{Discussion}

This article is protected by copyright. All rights reserved. 
For men and postmenopausal women, the effect of adiposity on CRC not explained by the included biomarkers was larger than the estimated indirect effect through all biomarkers. Pathways originating with CRP explained a small proportion of the adiposity-CRC association. In men, a small part of the adiposityCRC association was explained by adiposity-induced reduced levels of SHBG and testosterone.

To our knowledge, this is one of the first and largest studies that has attempted to quantify the mediating effects of multiple pathways on the adiposity-CRC association, while allowing for correlation between mediators and interrelation between pathways ${ }^{17}$. The causal mediation analysis approach was able to handle non-linear mediator-outcome associations and exposure-mediator or mediator-mediator interactions ${ }^{17}$. These are important advantages over other statistical methods that have traditionally been used to assess mediation ${ }^{28}$. The path-specific indirect effects were estimated using a sequential approach. An unverifiable assumption required by this approach is that the prespecified causal ordering of the pathways is true ${ }^{17}$. For this analysis, based on current evidence, we assumed that inflammatory and insulin signaling pathways preceded and possibly influenced SHBG and testosterone levels, while inflammatory pathway preceded dysregulated insulin signaling and glucose homeostasis ${ }^{9,14}$.

We made use of all the relevant biomarkers in the UK Biobank, but measurements were available for only a limited number of biomarkers, which was perhaps the most important limitation of this study. The observed mediating role for the pathways, or lack thereof, would have depended on how well the available biomarkers captured the biological characteristics of each pathway. For all participants, we used measurements for the biomarkers at one time point only. The differences in temporal variability and measurement quality of the biomarkers may have introduced varying degrees of information bias into our estimated associations and influenced the strength of the observed indirect effects. Overall, however, the biomarker measurements were of high quality (all CVs $<9 \%$ ) ${ }^{21,23,24}$ and, uniquely, were available in nearly all cohort participants.

Additionally, for all biomarkers, the ICCs calculated for the subgroup of participants who had measurements 
available for two time points (approximately four years apart) were $>0.55$, suggesting that single measurements provided a reasonable estimate of longer-term exposures. In the UK Biobank, height, weight, waist and hip circumference were measured following a published protocol to reduce measurement error ${ }^{20}$. In addition to BMI, we used waist circumference and waist-hip ratio as measures of adiposity, which may perform better in identifying individuals with obesity ${ }^{33}$. Our study did not provide evidence for an association between BMI and colorectal cancer risk in women, which was similar to a previous analysis of the UK Biobank data (hazard ratio (HR) for highest vs lowest quintile 1.11; 95\%CI, 0.89-1.38) ${ }^{34}$, but inconsistent with the results of a recent meta-analysis of studies investigating the association between BMI and colorectal cancer (summary RR for women per $5 \mathrm{~kg} / \mathrm{m}^{2} 1.05$; 95\%CI, 1.02-1.08) ${ }^{35}$. It is unclear why we found no association between BMI and colorectal cancer for women. One possibility is that a longer duration of follow-up and a larger number of cases may be required to detect the expected weaker (compared with men) BMI and colorectal cancer association. In support of this supposition, in the European Prospective Investigation into Cancer and Nutrition (EPIC) study after an average of 6.1 years of follow-up ( $n=1570$ cases), no association was found between BMI and colorectal cancer for women ${ }^{36}$. However, in a more recent EPIC study that included data after an average of 14.9 years of follow-up (n=6291 cases), the expected positive association between BMI and colorectal cancer for women was observed ${ }^{37}$.”

The sequential mediation analysis relied on assumptions of no unmeasured confounding of exposureoutcome, mediator-outcome, exposure-mediator, and mediator-mediator relations ${ }^{17}$. We had data on several potential confounders and took those into account in our analyses, but due to the observational nature of this study, residual confounding due to unmeasured or mismeasured confounders cannot be ruled out. For the adiposity-biomarker associations, this study was cross-sectional and, thus, prone to reverse causation. We 
excluded CRC cases diagnosed within the first year of follow-up to reduce the possibility of outcome having influenced adiposity or biomarker measurements.

Using causal mediation analysis in a nested case-control study within the Health Professionals Follow-up Study (HPFS), it was observed that a large proportion of the BMI-CRC association (OR per $3.6 \mathrm{~kg} / \mathrm{m}^{2} 1.40$; 95\%CI, 1.14-1.73) was explained by all inflammatory (CRP, interleukin-6, tumour necrosis factor receptor 2, and macrophage inhibitory cytokine-1) and metabolic (adiponectin, C-peptide, and soluble leptin receptor (sOB-R)) biomarkers (OR ${ }^{\mathrm{NIE}}$ 1.26; 95\%CI, 0.97-1.52) ${ }^{38}$. The authors repeated the mediation analysis separately for the two biomarker groups, assuming that the pathways were independent. Although the HPFS included more inflammatory biomarkers compared with ours, similar to our results, the observed indirect effect through inflammation was small (OR ${ }^{\mathrm{NIE}} 1.05$; 95\%CI, 0.96-1.14) ${ }^{38}$. Also, in a nested case-control study within the European Investigation into Cancer and Nutrition, adjusting for CRP only changed the RR for waist circumference-CRC association from 1.33 (95\%CI, 1.26-1.41) per $10 \mathrm{~cm}$ increment to 1.31 (95\%CI, 1.24-1.39) in men and from 1.20 (95\%CI, 1.14-1.25) to 1.19 (95\%CI, 1.14-1.25) in women ${ }^{39}$. Additional studies with a wider range of inflammatory biomarkers are needed to further explore the mediating role of inflammation in adiposity-CRC association.

The HPFS nested case-control study found a larger $\mathrm{OR}^{\mathrm{NIE}}$ for the metabolic biomarkers $\left(\mathrm{OR}^{\mathrm{NIE}} 1.24\right.$; 95\%CI, 0.92-1.55), which was mainly driven by adiponectin and sOB-R ${ }^{38}$. When the causal mediation analysis was repeated for individual biomarkers, weak to no mediating effect was observed for C-peptide (a biomarker for insulin signaling; $\mathrm{OR}^{\mathrm{NIE}} 1.05$; 95\%CI, 0.91-1.18) ${ }^{38}$. In a cohort study with 4,032 colon and 2,430 rectal cancer cases, the total BMI-colon (HR per $5 \mathrm{~kg} / \mathrm{m}^{2}$ 1.14; 95\%CI, 1.10-1.19) and -rectal cancer (HR 1.09; 95\%CI, 1.03-1.15) associations were weakly mediated by triglyceride-glucose index (TyG; a proxy for insulin resistance) ( $\mathrm{HR}^{\mathrm{NIE}} 1.03$; 95\%CI, 1.01-1.04 for colon and $\mathrm{HR}^{\mathrm{NIE}} 1.03$; 95\%CI, 1.01-1.05 for rectal cancer) ${ }^{40}$. The strength of the mediating effect was similar in men and women, and after excluding 
participants who did not have diabetes ${ }^{40}$. In our study, we did not observe any mediating effect through HbA1c. The observed indirect effect through TyG in the study by Fritz et al. may have been overestimated because the analysis did not allow for potential confounding by the preceding inflammatory pathway ${ }^{40}$. In our study, the estimated indirect effect through HbA1c excluded the possible influence of CRP on HbA1c, thus removed the confounding effect of CRP ${ }^{17}$. This issue, however, is unlikely to fully explain the null indirect effect for HbA1c we observed. In our study of men and postmenopausal women who at baseline did not have diabetes and did not report taking diabetes medication, there was no evidence for an association between HbA1c and CRC, regardless of adjustment for CRP. Similarly, a recent Mendelian randomization analysis found no evidence of an effect between genetically-predicted HbA1c concentration and colorectal cancer risk (OR per genetically determined 1 standard deviation (SD) increment 1.02; 95\%CI, 0.85 - 1.22) 41. Fritz et al. observed an association between TyG and colon (HR per 1 SD 1.07; 95\% CI, 1.03-1.10) and rectal (HR 1.09; 95\% CI, 1.04-1.14) cancers ${ }^{40}$. Although HbA1c is an established long-term biomarker of glucose and insulin metabolism ${ }^{42}$, it may not be the ideal biomarker of dysregulated insulin signaling on its own.

We are not aware of other studies that have investigated the mediating role of sex-steroid hormones in the adiposity-CRC association. We were also limited in exploring the role of this pathway fully because we did not have measures for estrogens. Our results provided evidence for a small role of adiposity-induced reduced levels of SHBG and testosterone in explaining the increased CRC risk in men with obesity. This observation is in line with the existing evidence on the potential protective role of androgens on CRC development ${ }^{43}$. It may also partly explain the stronger adiposity-CRC association observed in men because as found in ours and previous studies, adiposity is associated with reduced testosterone levels in men, but not in women ${ }^{43}$. 
In summary, increased CRP and reduced SHBG and testosterone levels explained a small proportion of the effect of adiposity on CRC in men. Increased CRP also had a small mediating effect on the association in postmenopausal women, comparable to the mediating effect observed in men. We did not observe any mediating effect for HbA1c. A large proportion of the effect of adiposity on CRC was not explained by the included biomarkers. These results suggest that pathways other than those captured here may also be important for understanding the mechanisms linking adiposity and CRC and for identifying targets for CRC prevention in individuals with obesity. Future studies with a wider range of biomarkers reflecting inflammatory status, impaired insulin signaling and hyperinsulinemia, and altered sex-steroid hormone levels in the obese state are needed to perform a comprehensive evaluation of the role of these pathways in explaining the effect of adiposity on CRC link. Proper causal mediation analysis approaches would allow such investigation, while appropriately accounting for correlations between biomarkers.

This article is protected by copyright. All rights reserved. 
Acknowledgements: This research was conducted using the UK Biobank Resource under application number 25897.

Disclaimer: Where authors are identified as personnel of the International Agency for Research on Cancer / World Health Organization, the authors alone are responsible for the views expressed in this article and they do not necessarily represent the decisions, policy or views of the International Agency for Research on Cancer / World Health Organization.

Financial Support: This work was supported by Australian National Health and Medical Research Council grants (1150591 to SGD and 1104975 to JAS); Melbourne Research Scholarship (to SGD), and the French National Cancer Institute (INCa, grant nr. 2017-127 to NM). MMB is the recipient of an Australian Research Council Discovery Early Career Award (project number DE190101326) funded by the Australian Government. The work reported in this paper was undertaken by SGD while hosted by the International Agency for Research on Cancer (IARC) and funded partially by a PhD Scholarship by IARC. The funders did not influence the design of the study, analysis or interpretation of the data, the writing of this report or the decision to publish.

Conflict of interest: The authors declare no potential conflicts of interest.

This article is protected by copyright. All rights reserved. 


\section{References}

1. Bray F, Ferlay J, Soerjomataram I, Siegel RL, Torre LA, Jemal A. Global cancer statistics 2018: GLOBOCAN estimates of incidence and mortality worldwide for 36 cancers in 185 countries. CA Cancer J Clin 2018; 68: 394-424.

2. Arnold M, Sierra MS, Laversanne M, Soerjomataram I, Jemal A, Bray F. Global patterns and trends in colorectal cancer incidence and mortality. Gut 2017; 66: 683-91.

3. Lauby-Secretan B, Scoccianti C, Loomis D, Grosse Y, Bianchini F, Straif K, International Agency for Research on Cancer Handbook Working G. Body Fatness and Cancer--Viewpoint of the IARC Working Group. $N$ Engl J Med 2016; 375: 794-8.

4. Ma Y, Yang Y, Wang F, Zhang P, Shi C, Zou Y, Qin H. Obesity and risk of colorectal cancer: a systematic review of prospective studies. PLoS One 2013; 8: e53916.

5. Arnold M, Pandeya N, Byrnes G, Renehan PAG, Stevens GA, Ezzati PM, Ferlay J, Miranda JJ, Romieu I, Dikshit R, Forman D, Soerjomataram I. Global burden of cancer attributable to high body-mass index in 2012: a population-based study. Lancet Oncol 2015; 16: 36-46.

6. N. C. D. Risk Factor Collaboration. Trends in adult body-mass index in 200 countries from 1975 to 2014: a pooled analysis of 1698 population-based measurement studies with 19.2 million participants. Lancet 2016; 387: 1377-96.

7. N. C. D. Risk Factor Collaboration. Worldwide trends in body-mass index, underweight, overweight, and obesity from 1975 to 2016: a pooled analysis of 2416 population-based measurement studies in 128.9 million children, adolescents, and adults. Lancet 2017; 390: 2627-42.

8. Murphy N, Jenab M, Gunter MJ. Adiposity and gastrointestinal cancers: epidemiology, mechanisms and future directions. Nat Rev Gastroenterol Hepatol 2018; 15: 659-70.

9. Hardy OT, Czech MP, Corvera S. What causes the insulin resistance underlying obesity? Curr Opin Endocrinol Diabetes Obes 2012; 19: 81-7. (4): 181-90.

10. Pelusi C, Pasquali R. The Significance of Low Testosterone Levels in Obese Men. Curr Obes Rep 2012; 1

11. Gerard C, Brown KA. Obesity and breast cancer - Role of estrogens and the molecular underpinnings of aromatase regulation in breast adipose tissue. Mol Cell Endocrinol 2017; 466: 15-30.

12. Renehan AG, Frystyk J, Flyvbjerg A. Obesity and cancer risk: the role of the insulin-IGF axis. Trends Endocrinol Metab 2006; 17: 328-36.

13. Pollak M. Insulin and insulin-like growth factor signalling in neoplasia. Nat Rev Cancer 2008; 8: 915-28. 
14. Iyengar NM, Gucalp A, Dannenberg AJ, Hudis CA. Obesity and Cancer Mechanisms: Tumor Microenvironment and Inflammation. J Clin Oncol 2016; 34: 4270-6. 8: R1-R9.

15. Gambineri A, Pelusi C. Sex hormones, obesity and type 2 diabetes: is there a link? Endocr Connect 2019;

16. Johnson JR, Lacey JV, Jr., Lazovich D, Geller MA, Schairer C, Schatzkin A, Flood A. Menopausal hormone therapy and risk of colorectal cancer. Cancer Epidemiol Biomarkers Prev 2009; 18: 196-203. 2: 95-115.

17. VanderWeele TJ, Vansteelandt S. Mediation Analysis with Multiple Mediators. Epidemiol Methods 2014;

18. Sherwani SI, Khan HA, Ekhzaimy A, Masood A, Sakharkar MK. Significance of HbA1c Test in Diagnosis and Prognosis of Diabetic Patients. Biomark Insights 2016; 11: 95-104.

19. Allen N, Sudlow C, Downey P, Peakman T, Danesh J, Elliott P, Gallacher J, Green J, Matthews P, Pell J, Sprosen T, Collins R, Biobank U. UK Biobank: Current status and what it means for epidemiology. Health Policy Techn 2012; 1: 123-6.

20. UK Biobank. Body Composition Measurement: Version 1.0, 2011.

21. UK Biobank. Biomarker assay quality procedures: approaches used to minimise systematic and random errors (and the wider epidemiological implications): Version 1.2, 2019.

22. Hosgood HD, Gunter MJ, Murphy N, Rohan TE, Strickler HD. The Relation of Obesity-Related Hormonal and Cytokine Levels With Multiple Myeloma and Non-Hodgkin Lymphoma. Front Oncol 2018; 8: 103.

23. UK Biobank. UK Biobank Biomarker Enhancement Project Companion Document to Accompany HbA1c Biomarker Data: Version 1.0, 2018.

24. UK Biobank. UK Biobank Biomarker Project Companion Document to Accompany Serum Biomarker Data: Version 1.0, 2019.

25. de Ronde W, van der Schouw YT, Pols HA, Gooren LJ, Muller M, Grobbee DE, de Jong FH. Calculation of bioavailable and free testosterone in men: a comparison of 5 published algorithms. Clin Chem 2006; 52: 1777-84.

26. World Health Organization. Waist Circumference and Waist-Hip Ratio: Report of a WHO Expert Consultation. https://apps.who.int/iris/bitstream/handle/10665/44583/9789241501491_eng.pdf?sequence=1; 2008.

27. Lin SH, Young JG, Logan R, VanderWeele TJ. Mediation analysis for a survival outcome with timevarying exposures, mediators, and confounders. Stat Med 2017; 36: 4153-66.

28. VanderWeele TJ. Mediation Analysis: A Practitioner's Guide. Annu Rev Public Health 2016; 37: 17-32.

29. Cummings P. Methods for estimating adjusted risk ratios. Stata J 2009; 9: 175-96. 
30. Tilling K, Williamson EJ, Spratt M, Sterne JA, Carpenter JR. Appropriate inclusion of interactions was needed to avoid bias in multiple imputation. J Clin Epidemiol 2016; 80: 107-15.

31. Schomaker M, Heumann H. Bootstrap inference when using multiple imputation. Statistics in Medicine 2018; 37: 2252-66.

32. StataCorp. 2017. Stata Statistical Software: Release 15. College Station, TX: StataCorp LLC.

33. Nimptsch K, Pischon T. Obesity Biomarkers, Metabolism and Risk of Cancer: An Epidemiological Perspective. Recent Results Cancer Res 2016; 208: 199-217.

34. Ortega LS, Bradbury KE, Cross AJ, Morris JS, Gunter MJ, Murphy N. A Prospective Investigation of Body Size, Body Fat Composition and Colorectal Cancer Risk in the UK Biobank. Sci Rep 2017; 7: 17807.

35. World Cancer Research Fund International/American Institute for Cancer Research, Continuous Update Project Report: Diet, Nutrition, Physical Activity and Colorectal Cancer, 2017.

36. Pischon T, Lahmann PH, Boeing H, Friedenreich C, Norat T, Tjonneland A, Halkjaer J, Overvad K, Clavel-Chapelon F, Boutron-Ruault MC, Guernec G, Bergmann MM, Linseisen J, Becker N, Trichopoulou A, Trichopoulos D, Sieri S, Palli D, Tumino R, Vineis P, Panico S, Peeters PH, Bueno-de-Mesquita HB, Boshuizen HC, Van Guelpen B, Palmqvist R, Berglund G, Gonzalez CA, Dorronsoro M, Barricarte A, Navarro C, Martinez C, Quiros JR, Roddam A, Allen N, Bingham S, Khaw KT, Ferrari P, Kaaks R, Slimani N, Riboli E. Body size and risk of colon and rectal cancer in the European Prospective Investigation Into Cancer and Nutrition (EPIC). $J$ Natl Cancer Inst 2006; 98: 920-31.

37. Murphy N, Ward HA, Jenab M, Rothwell JA, Boutron-Ruault MC, Carbonnel F, Kvaskoff M, Kaaks R, Kuhn T, Boeing H, Aleksandrova K, Weiderpass E, Skeie G, Borch KB, Tjonneland A, Kyro C, Overvad K, Dahm CC, Jakszyn P, Sanchez MJ, Gil L, Huerta JM, Barricarte A, Quiros JR, Khaw KT, Wareham N, Bradbury KE, Trichopoulou A, La Vecchia C, Karakatsani A, Palli D, Grioni S, Tumino R, Fasanelli F, Panico S, Bueno-deMesquita B, Peeters PH, Gylling B, Myte R, Jirstrom K, Berntsson J, Xue X, Riboli E, Cross AJ, Gunter MJ. Heterogeneity of Colorectal Cancer Risk Factors by Anatomical Subsite in 10 European Countries: A Multinational Cohort Study. Clin Gastroenterol Hepatol 2019; 17: 1323-31 e6.

38. Petimar J, Tabung FK, Valeri L, Rosner B, Chan AT, Smith-Warner SA, Giovannucci EL. Mediation of associations between adiposity and colorectal cancer risk by inflammatory and metabolic biomarkers. Int $J$ Cancer 2019; 144: 2945-53.

39. Aleksandrova K, Drogan D, Boeing H, Jenab M, Bas Bueno-de-Mesquita H, Jansen E, van Duijnhoven FJ, Rinaldi S, Fedirko V, Romieu I, Kaaks R, Riboli E, Gunter MJ, Romaguera D, Westhpal S, Overvad K, Tjonneland A, Halkjaer J, Boutron-Ruault MC, Clavel-Chapelon F, Lukanova A, Trichopoulou A, Trichopoulos D, Vidalis P, Panico S, Agnoli C, Palli D, Tumino R, Vineis P, Buckland G, Sanchez-Cruz JJ, Dorronsoro M, Diaz MJ, Barricarte A, Ramon Quiros J, Peeters PH, May AM, Hallmans G, Palmqvist R, Crowe FL, Khaw KT, Wareham N, Pischon T. Adiposity, mediating biomarkers and risk of colon cancer in the European prospective investigation into cancer and nutrition study. Int J Cancer 2014; 134: 612-21. 
40. Fritz J, Bjorge T, Nagel G, Manjer J, Engeland A, Haggstrom C, Concin H, Teleka S, Tretli S, Gylling B, Lang A, Stattin P, Stocks T, Ulmer H. The triglyceride-glucose index as a measure of insulin resistance and risk of obesity-related cancers. Int J Epidemiol 2019.

41. Cornish AJ, Law PJ, Timofeeva M, Palin K, Farrington SM, Palles C, Jenkins MA, Casey G, Brenner H, Chang-Claude J, Hoffmeister M, Kirac I, Maughan T, Brezina S, Gsur A, Cheadle JP, Aaltonen LA, Tomlinson I, Dunlop MG, Houlston RS. Modifiable pathways for colorectal cancer: a mendelian randomisation analysis. Lancet Gastroenterol Hepatol 2019.

42. Xu J, Ye Y, Wu H, Duerksen-Hughes P, Zhang H, Li P, Huang J, Yang J, Wu Y, Xia D. Association between markers of glucose metabolism and risk of colorectal cancer. BMJ Open 2016; 6: e011430.

43. Lin JH, Giovannucci E. Sex hormones and colorectal cancer: what have we learned so far? J Natl Cancer Inst 2010; 102: 1746-7. 


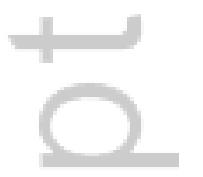

This article is protected by copyright. All rights reserved. 
Tables

Table 1 - Baseline Characteristics of men and women eligible for the study by categories of waist circumference

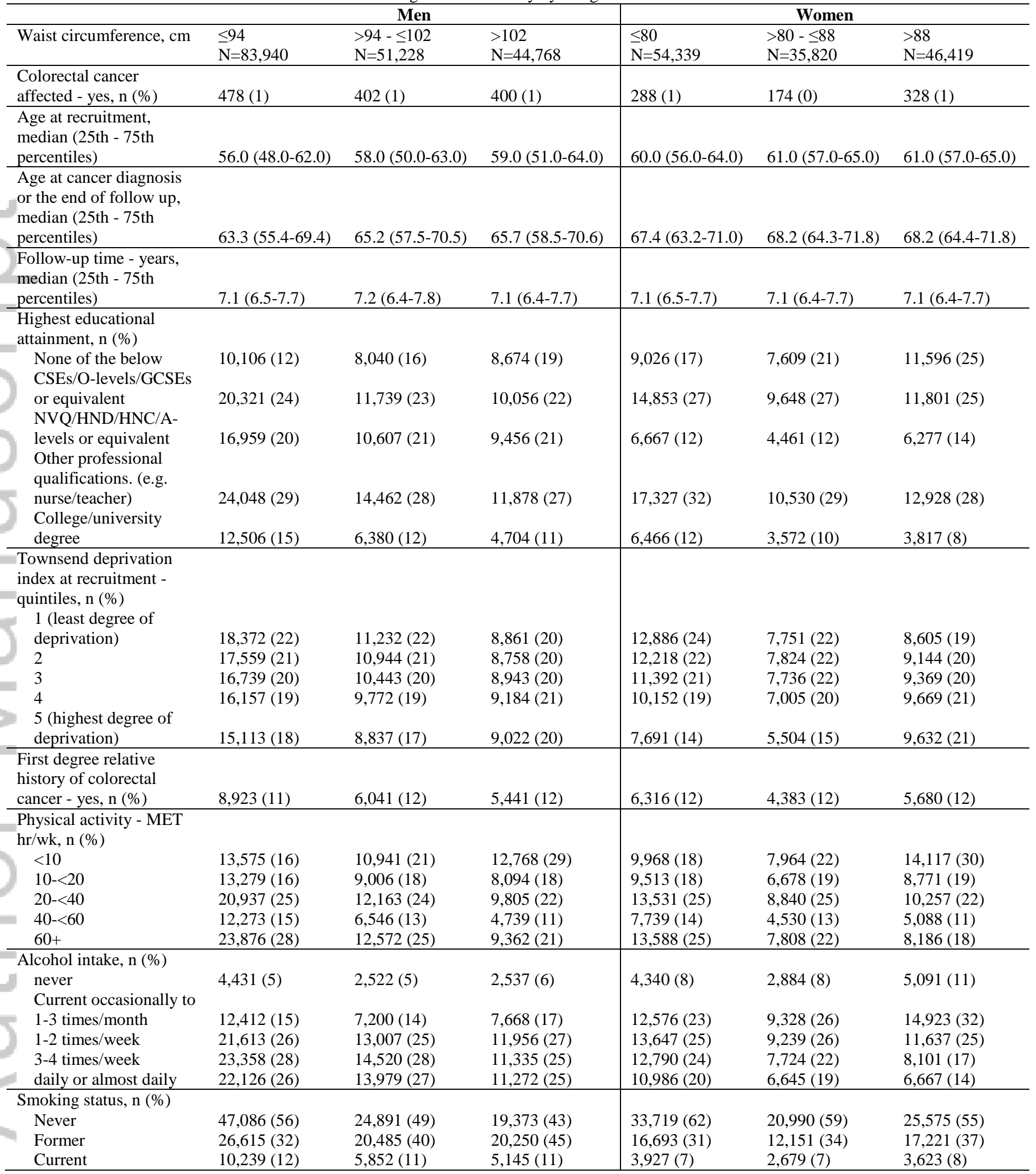


Total red and processed

meat intake - times/week,

median (25th - 75th

$3.5(2.5-5.0)$

$4.0(2.5-5.0)$

$4.5(2.5-5.5)$

$2.5(2.0-4.0)$

$2.5(2.0-4.5)$

$3.0(2.0-4.5)$

Regular aspirin or

ibuprofen use - yes, n (\%)

Number of live births,

median (25th - 75th

percentiles)

$18,700(22)$

$14,187(28)$

14,657 (33)

$10,656(20)$

$8,427(24)$

$12,954(28)$

$2.0(1.0-2.0)$

$2.0(1.0-3.0)$

$2.0(1.0-3.0)$

This article is protected by copyright. All rights reserved. 


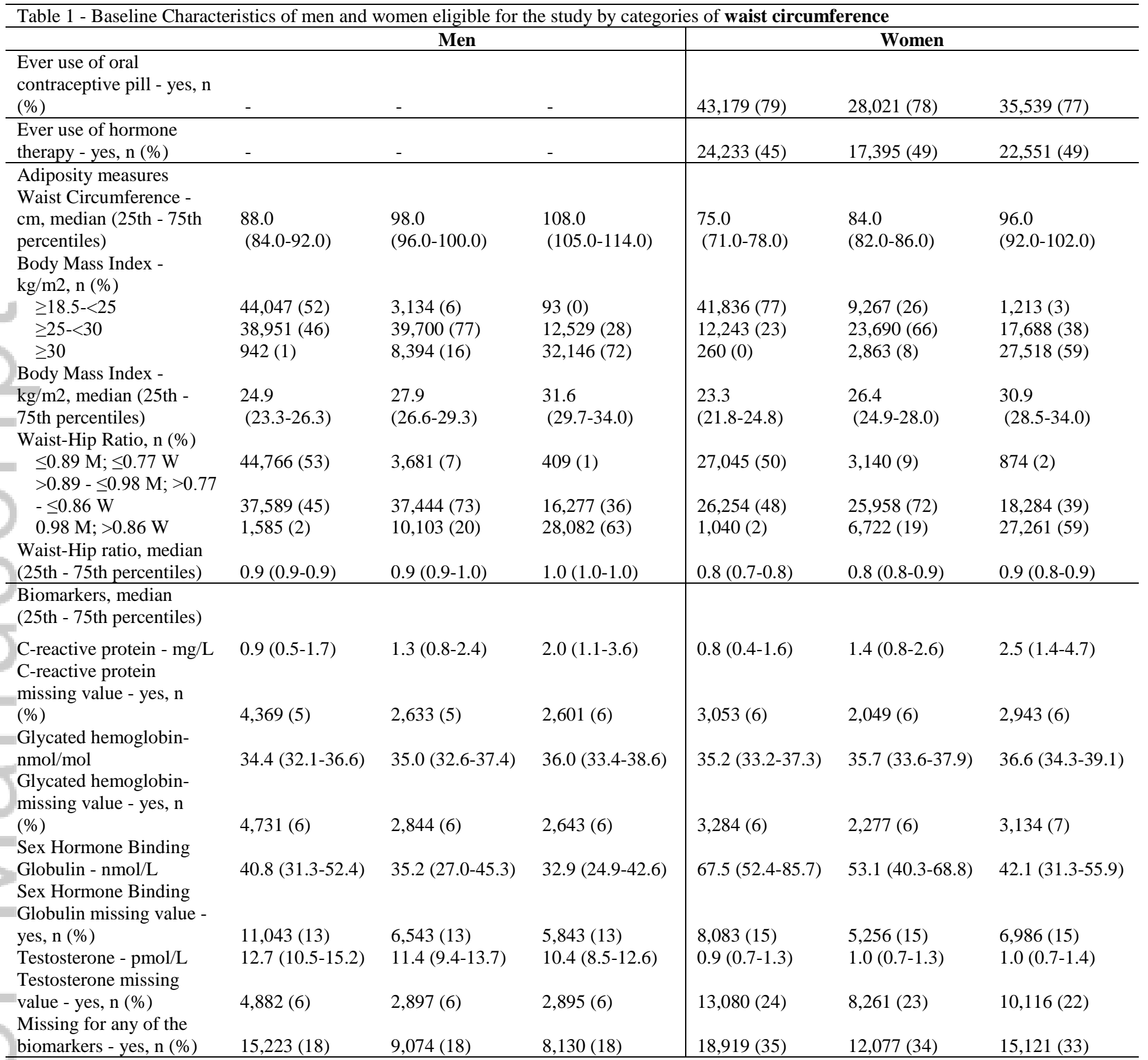

CSE Certificate of Secondary Education; GCSE General Certificate of Secondary Education; NVQ National Vocational Education; HND Higher National Diploma; HNC Higher National Certificate; MET Metabolic Equivalent of Task; W women; M men

Baseline Characteristics of men and women eligible for the study by categories of body mass index and waist-hip ratio are presented in Supplementary Table 1 and Supplementary Table 2 respectively. 
Table 2 - Association between adiposity measures and biomarkers; multiple imputation analyses excluding women in the middle category of each adiposity measure

\begin{tabular}{|c|c|c|c|c|c|c|c|c|c|c|}
\hline & & & & Ratio o & ometr & ans (95\% & onfide & interv & & \\
\hline & & & ircumfe & ence & & & & & t-Hip Ra & \\
\hline & & & vs. $\leq 94$ & & & Mass In & & & 8 vs. $\leq 89$ & \\
\hline & Biomarker & & vs. $\leq 80$ & & & s. $<25 \mathrm{~kg}$ & & & 6 vs. $\leq 77$ & \\
\hline & & & $=128,708$ & & & $=88,756$ & & & $=88,626$ & \\
\hline & CRP & 2.00 & (1.97 to & 2.02) & 2.31 & (2.28 to & 2.34) & 2.10 & (2.07 to & 2.13) \\
\hline 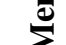 & $\mathrm{HbA1C}$ & 1.04 & (1.04 to & 1.05) & 1.05 & (1.05 to & 1.05) & 1.05 & (1.05 to & 1.05) \\
\hline & SHBG & 0.79 & (0.79 to & 0.79) & 0.72 & (0.72 to & $0.73)$ & 0.77 & (0.76 to & $0.77)$ \\
\hline & Testosterone & 0.83 & (0.82 to & 0.83) & 0.80 & ( 0.80 to & $0.80)$ & 0.83 & (0.83 to & $0.84)$ \\
\hline & & & $=100,758$ & & & $=82,957$ & & & $=66,082$ & \\
\hline & CRP & 2.64 & (2.60 to & 2.67) & 3.19 & (3.15 to & $3.24)$ & 2.19 & (2.16 to & 2.23) \\
\hline$\Xi$ & $\mathrm{HbA} 1 \mathrm{C}$ & 1.04 & (1.04 to & 1.04) & 1.04 & (1.04 to & 1.04) & 1.05 & (1.05 to & 1.05) \\
\hline & SHBG & 0.63 & (0.63 to & 0.63) & 0.61 & (0.60 to & $0.61)$ & 0.63 & (0.62 to & $0.63)$ \\
\hline & Testosterone & 1.08 & (1.07 to & 1.09) & 1.12 & (1.11 to & 1.13) & 1.04 & (1.03 to & 1.04) \\
\hline
\end{tabular}

Results from complete case analysis are provided in Supplementary Table 5.

Abbreviations: CRP C-reactive protein; HbA1c glycated hemoglobin; SHBG sex-hormone-binding globulin

Missing biomarker data were multiply imputed (complete case analysis results are presented in Supplementary Table 4).

Models were adjusted for age at recruitment, education, Townsend deprivation index at recruitment, first-degree relative history of colorectal cancer, physical activity, alcohol intake, smoking status, red and processed meat intake, and regular use of aspirin or ibuprofen. Models for women were additionally adjusted for the number of live births, ever use of oral contraceptive pill, and ever use of hormone therapy. 
Table 3 - Estimated odds ratios and 95\% confidence intervals for the association between adiposity measures (exposure) or biomarkers (mediators) and colorectal cancer (outcome) in men and women; multiple imputation analysis

\begin{tabular}{|c|c|c|c|c|c|c|c|c|}
\hline & \multicolumn{4}{|c|}{ Men } & \multicolumn{4}{|c|}{ Women } \\
\hline Waist Circumference, cm & \multicolumn{3}{|c|}{$>102$ vs $<94$} & & \multicolumn{3}{|c|}{$>88$ vs $<80$} & \\
\hline OR (95\% CI) & 1.38 & (1.20 to & 1.59) & & 1.28 & (1.08 to & $1.51)$ & \\
\hline Body Mass Index, kg/m² & \multicolumn{3}{|c|}{$\geq 30$ vs $<25$} & & \multicolumn{3}{|c|}{$\geq 30$ vs $<25$} & \\
\hline OR (95\% CI) & 1.37 & (1.16 to & $1.62)$ & & 1.11 & (0.91 to & 1.36) & \\
\hline Waist-Hip ratio & \multicolumn{3}{|c|}{$>0.98$ vs $<0.89$} & & \multicolumn{3}{|c|}{$>0.86$ vs $<0.77$} & \\
\hline OR $(95 \% \mathrm{CI})$ & 1.42 & (1.19 to & $1.70)$ & & 1.33 & (1.07 to & 1.65) & \\
\hline Biomarker & \multicolumn{3}{|c|}{$\begin{array}{l}\text { Per doubling } \\
\text { concentration }\end{array}$} & $\begin{array}{c}\text { P value } \\
\text { for } \\
\text { evidence } \\
\text { against } \\
\text { linearity }\end{array}$ & \multicolumn{3}{|c|}{$\begin{array}{l}\text { Per doubling } \\
\text { concentration }\end{array}$} & $\begin{array}{c}\text { P value } \\
\text { for } \\
\text { evidence } \\
\text { against } \\
\text { linearity }\end{array}$ \\
\hline Model 1 & 1.07 & (1.02 to & 1.11) & 0.02 & 1.03 & (0.98 to & 1.09) & 0.75 \\
\hline $\begin{array}{l}\text { HbA1C } \\
\text { Model } 1\end{array}$ & 0.85 & (0.61 to & 1.19) & & 1.08 & (0.67 to & 1.75) & \\
\hline $\begin{array}{l}\text { Model } 2 \\
\text { (additionally adjusted for CRP) }\end{array}$ & 0.82 & (0.58 to & 1.14) & 0.15 & 1.06 & (0.66 to & 1.71) & 0.75 \\
\hline $\begin{array}{l}\text { SHBG } \\
\text { Model } 1\end{array}$ & 0.92 & (0.82 to & 1.02) & & 1.01 & (0.89 to & 1.15) & \\
\hline $\begin{array}{l}\text { Model } 2 \\
\text { (additionally adjusted for CRP and HbA1c) }\end{array}$ & 0.92 & (0.82 to & 1.03) & 0.20 & 1.03 & (0.90 to & 1.18) & 0.00 \\
\hline $\begin{array}{l}\text { Testosterone } \\
\text { Model } 1\end{array}$ & 0.91 & ( 0.80 to & 1.04) & & 1.00 & (0.88 to & 1.13) & \\
\hline $\begin{array}{l}\text { Model } 2 \\
\text { (additionally adjusted for CRP and HbA1c) }\end{array}$ & 0.92 & (0.81 to & 1.05) & 0.56 & 1.00 & (0.88 to & 1.13) & 0.19 \\
\hline
\end{tabular}

Results from complete case analysis are provided in Supplementary Table 6.

Abbreviations: CRP C-reactive protein; HbA1c glycated hemoglobin; SHBG sex-hormone-binding globulin

Missing biomarker data were multiply imputed (complete case analysis results are presented in Supplementary Table 5).

Models were adjusted for age at recruitment, education, Townsend deprivation index at recruitment, first-degree relative history of colorectal cancer, physical activity, alcohol intake, smoking status, red and processed meat intake, and regular use of aspirin or ibuprofen. Models for women were additionally adjusted for the number of live births, ever use of oral contraceptive pill, and ever use of hormone therapy. Model 1 for biomarkers were additionally adjusted for waist circumference. 
Table 4- Estimated natural direct and indirect effects using sequential mediation analysis for the association between adiposity measures and colorectal cancer for men and women, analyses exclude participants categorized as overweight and do not include any interaction terms between adiposity measures and biomarkers (exposure-mediator interactions) or biomarkers (mediatormediator interactions) - multiple imputation analysis

\begin{tabular}{|c|c|c|c|c|c|c|c|}
\hline & \multirow[b]{2}{*}{ Effect } & \multicolumn{6}{|c|}{ Risk Ratio (95\% CI) } \\
\hline & & \multicolumn{3}{|c|}{ Men } & \multicolumn{3}{|c|}{ Women } \\
\hline \multirow{7}{*}{$\begin{array}{c}\text { Waist } \\
\text { Circumference } \\
>102 \text { vs. } \leq 94 \mathrm{~cm} \mathrm{M} \\
>88 \text { vs. } \leq 80 \mathrm{~cm} \mathrm{~F}\end{array}$} & No. Colorectal Cancer affected/ No. unaffected & \multicolumn{3}{|c|}{$878 / 127,830$} & \multicolumn{3}{|c|}{$616 / 100,142$} \\
\hline & Total effect & 1.37 & (1.19 to & 1.58) & 1.27 & (1.07 to & $1.50)$ \\
\hline & Natural indirect effect through all the mediators & 1.08 & (1.01 to & 1.16) & 1.08 & (0.95 to & 1.22) \\
\hline & Natural indirect effect through CRP & 1.06 & (1.01 to & 1.11) & 1.08 & (0.99 to & 1.17) \\
\hline & $\begin{array}{l}\text { Natural indirect effect through HbA1C, excluding } \\
\text { the influence of CRP }\end{array}$ & 0.99 & $(0.97$ to & 1.01) & 1.00 & (0.98 to & 1.02) \\
\hline & $\begin{array}{l}\text { Natural indirect effect through SHBG and } \\
\text { testosterone, excluding the influence of CRP and } \\
\text { HbA1c }\end{array}$ & 1.03 & (0.99 to & 1.08) & 1.00 & $(0.92$ to & 1.09) \\
\hline & $\begin{array}{l}\text { Natural direct effect not through any of the } \\
\text { mediators }\end{array}$ & 1.26 & (1.09 to & 1.47) & 1.18 & $(0.96$ to & $1.45)$ \\
\hline \multirow{7}{*}{$\begin{array}{l}\text { Body Mass Index } \\
\geq 30 \text { vs. }<25 \mathrm{~kg} / \mathrm{m} 2\end{array}$} & No. Colorectal Cancer affected/ No. unaffected & \multicolumn{3}{|c|}{$614 / 88,142$} & \multicolumn{3}{|c|}{$451 / 82,506$} \\
\hline & Total effect & 1.36 & (1.15 to & 1.60) & 1.12 & $(0.92$ to & 1.38) \\
\hline & Natural indirect effect through all the mediators & 1.11 & (1.00 to & 1.22) & - & - & - \\
\hline & Natural indirect effect through CRP & 1.06 & (0.99 to & 1.13) & - & - & - \\
\hline & $\begin{array}{l}\text { Natural indirect effect through HbA1C, excluding } \\
\text { the influence of CRP }\end{array}$ & 0.99 & $(0.96$ to & 1.02) & - & - & - \\
\hline & $\begin{array}{l}\text { Natural indirect effect through SHBG and } \\
\text { testosterone, excluding the influence of CRP and } \\
\text { HbA1c }\end{array}$ & 1.05 & (0.99 to & 1.12) & - & - & - \\
\hline & $\begin{array}{l}\text { Natural direct effect not through any of the } \\
\text { mediators }\end{array}$ & 1.23 & (1.02 to & 1.48) & - & - & - \\
\hline \multirow{7}{*}{$\begin{array}{l}\text { Waist-Hip Ratio } \\
>0.98 \text { vs. }<0.89 \mathrm{M} \\
>0.86 \text { vs. } \leq 0.77 \mathrm{~F}\end{array}$} & No. Colorectal Cancer affected/ No. unaffected & \multicolumn{3}{|c|}{$600 / 88,026$} & \multicolumn{3}{|c|}{$378 / 65,704$} \\
\hline & Total effect & 1.31 & (1.09 to & 1.58) & 1.33 & (1.06 to & 1.66) \\
\hline & Natural indirect effect through all the mediators & 1.12 & (1.03 to & 1.23) & 1.10 & $(0.96$ to & 1.26) \\
\hline & Natural indirect effect through CRP & 1.07 & (1.00 to & 1.14) & 1.09 & (1.00 to & 1.19) \\
\hline & $\begin{array}{l}\text { Natural indirect effect through HbA1C, excluding } \\
\text { the influence of CRP }\end{array}$ & 0.99 & $(0.96$ to & 1.02) & 1.01 & $(0.97$ to & 1.05) \\
\hline & $\begin{array}{l}\text { Natural indirect effect through SHBG and } \\
\text { testosterone, excluding the influence of CRP and } \\
\text { HbA1c }\end{array}$ & 1.06 & $(1.01$ to & 1.11) & 1.00 & (0.91 to & 1.11) \\
\hline & $\begin{array}{l}\text { Natural direct effect not through any of the } \\
\text { mediators }\end{array}$ & 1.17 & (0.95 to & 1.44) & 1.20 & (0.92 to & 1.57) \\
\hline
\end{tabular}

Results from complete case analysis are provided in Supplementary Table 7.

Abbreviations: CRP C-reactive protein; HbA1c glycated hemoglobin; SHBG sex-hormone-binding globulin

Models were adjusted for age at recruitment, education, Townsend deprivation index at recruitment, first-degree relative history of colorectal cancer, physical activity, alcohol intake, smoking status, red and processed meat intake, and regular use of aspirin or ibuprofen. Models for women were additionally adjusted for the number of live births, ever use of oral contraceptives, and ever use of hormone therapy. 
Figure Legends

Figure 1a- Flow diagram demonstrating the selection process of men in the UK Biobank for the present study

Figure 1b- Flow diagram demonstrating the selection process of women in the UK Biobank for the present study

Figure 2 - Schematic interpretation of the estimated total, natural direct, and natural indirect effects In this study, C-reactive protein (CRP) was used as a general marker for inflammation, HbA1C as a marker for glycemic control and a correlate of insulin resistance, and sex-hormone-binding globulin (SHBG) and testosterone treated jointly as makers of the sex-steroid hormone pathway. Waist circumference, BMI, and waist-hip ratio were used as proxy measures for adiposity in separate sets of analyses. In each provided outcome model needed to estimate the effects, $y$ is the outcome (colorectal cancer incidence), $a$ is the exposure (each adiposity measure comparing obese vs normal), c confounders, m1 CRP, m2 HbA1c, m3a SHBG, and m3b testosterone.

This article is protected by copyright. All rights reserved. 


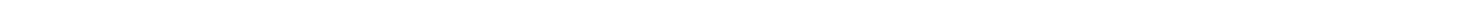




\section{5}

Excluded:

Withdrew consent; $n=2$

Diagnosed with cancer before baseline; $n=17,038$

Had less than one year follow-up time; $\mathrm{n}=229$

Died or were lost to follow up before the end of follow-up; $n=2,138$ Body size measurements (weight, height, waist circumference) not recorded at baselines; $\mathrm{n}=1,333$ Body mass index $<18.5 \mathrm{~kg} / \mathrm{m}^{2}$; $\mathrm{n}=1,888$

Unknown diabetes status at baseline; $n=886$

Diabetic at baseline, users of diabetes medication at baseline; $n=9,320$ Unknown menopause status at baseline and 55 years old or younger at baseline; $n=17,637$

Premenopausal at baseline; $n=60,072$

Hormone therapy user at baseline; $\mathrm{n}=11,020$

Hormonal contraceptive user at baseline; $n=131$

Missing data for confounders; $n=14,384(9.6 \%)$
With colorectal cancer diagnosed after baseline $\mathrm{n}=1,534$

\section{$\mathrm{n}=271,865$}

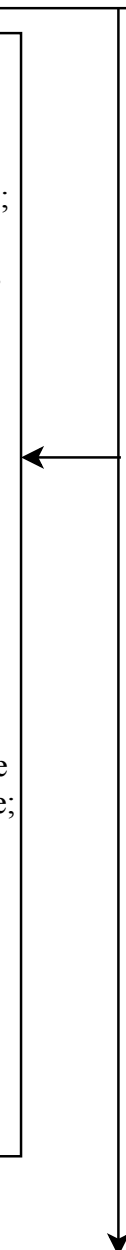

ithout colorectal cancer available for analysis $\mathrm{n}=135,788$
Missing data for any of the biomarkers of interest; $n=43,839$ $(34 \%)$ 
Figure 2 - Schematic interpretation of the estimated total, natural direct, and natural indirect effects

In this study, C-reactive protein (CRP) was used as a general marker for inflammation, HbAlC as a marker for glycemic control and a correlate of insulin resistance, an sex-hormone-binding globulin (SHBG) and testosterone treated jointly as makers of the sex-steroid hormone pathway. Waist circumference, BMI, and waist-hip ratio were used as proxy measures for adiposity in separate sets of analyses. In each provided outcome model needed to estimate the effecys,is the outcome (colorectal cancer incidence), $\boldsymbol{a}$ is the exposure (each adiposity measure comparing obese vs normal) $\boldsymbol{c}$ confounders, $\boldsymbol{m} \mathbf{1}$ CRP, $\boldsymbol{m} \boldsymbol{2} \mathrm{HbA} 1 \mathrm{c}, \boldsymbol{m} \mathbf{3} \boldsymbol{a}$ SHBG, and $\boldsymbol{m} \mathbf{3} \boldsymbol{b}$ testosterone. The total effect of adiposity and CRC

This effect captures the adiposity-CRC association through the included biomarkers (the indirect effect) and not through the included biomarkers (referred to as the direct effect)

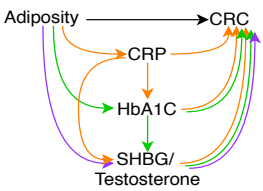

Required models for the outcome

The natural direct effect (NDE) not through the included biomarkers This effect captures the adiposity-CRC association not through any of the included biomarkers (the solid arrow).

logistic y a c

Required models for the outcome logistic y a c m1 m2 m3a m3b \& logistic y a c

The natural indirect effect through all included biomarkers

This effect captures the adiposity-CRC association through all the included biomarkers (the solid arrows)

This effect captures the effect of adiposity-induced changes in CRP levels on CRC risk directly (1), or indirectly through influencing HbAlC (2) or SHBG and testosterone (3) levels.

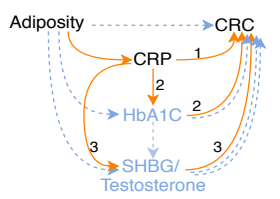

Required models for the outcome

logistic y a c m1 \& logistic y a c

The natural indirect effect through $\mathrm{HbA1C}$ beyond the influence of $\mathrm{C}$ reactive protein

This effect $(C)$ is estimated as the difference between the indirect effect through CRP and HbAlC (A) and the indirect effect through CRP (B). It thus excludes the possible influence of CRP on $\mathrm{HbA1C}$ levels and captures the effect of adiposity-induced changes in $\mathrm{HbA1C}$ on CRC risk directly (1), or indirectly through influencing SHBG and testosterone levels (2)

Required models for the outcome The natural indirect effect through sex-hormone-binding globulin and testosterone beyond the influences of $\mathrm{C}$-reactive protein and $\mathrm{HbA1C}$ This effect $(\mathrm{C})$ is estimated as the difference between the indirect effect through $\mathrm{CRP}, \mathrm{HbAlC}$, and SHBG and testosterone (A) and the indirect effect through $\mathrm{CRP}$ and $\mathrm{HbA} 1 \mathrm{C}$ (B). It thus excludes the possible influence of CRP or $\mathrm{HbAlC}$ on SHBG and testosterone levels and captures the effect adiposity-induced changes in SHBG and testosterone have on CRC risk (1).

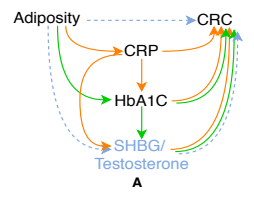

logistic y a c $\mathrm{m} 1 \mathrm{~m} 2$ \& logistic y $a c$

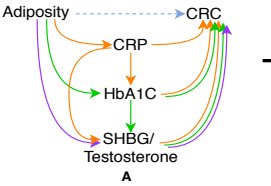

logistic y a c $m 1 \mathrm{~m} 2 \mathrm{~m} 3$ a $\mathrm{m} 3 \mathrm{~b} \quad$ logistic y a c $\mathrm{ml} \mathrm{m} 2$
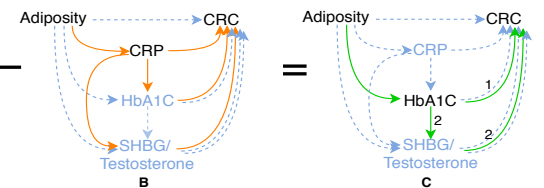

logistic y a $\mathrm{c} \mathrm{ml}$ \& logistic y a $c$
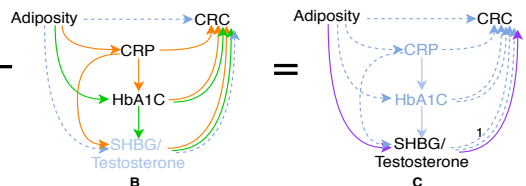
Novelty \& Impact Statement: IJC-20-0035.R1

Mechanisms underlying the association between adiposity and colorectal cancer (CRC) are not well understood. Here, using UK Biobank data and sequential mediation analysis, the authors quantified the effects of C-reactive protein, hemoglobin-A1c, sex-hormone-binding globulin (SHBG), and testosterone on the adiposity-CRC association in men and postmenopausal women. Analyses show that, in both men and women, increased CRP had small mediating effects on adiposity and CRC. In men, a small proportion of the effect was further explained by reduced SHBG and testosterone levels. The results suggest that pathways other than those captured in the study are important for understanding the adiposity-CRC link. 


\section{University Library}

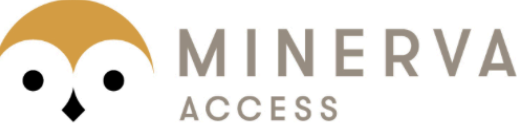

A gateway to Melbourne's research publications

Minerva Access is the Institutional Repository of The University of Melbourne

\section{Author/s:}

Dashti, SG;Viallon, V;Simpson, JA;Karahalios, A;Moreno-Betancur, M;English, DR;Gunter, MJ;Murphy, N

Title:

Explaining the link between adiposity and colorectal cancer risk in men and postmenopausal women in the UK Biobank: A sequential causal mediation analysis

Date:

2020-04-13

\section{Citation:}

Dashti, S. G., Viallon, V., Simpson, J. A., Karahalios, A., Moreno-Betancur, M., English, D. R., Gunter, M. J. \& Murphy, N. (2020). Explaining the link between adiposity and colorectal cancer risk in men and postmenopausal women in the UK Biobank: A sequential causal mediation analysis. INTERNATIONAL JOURNAL OF CANCER, 147 (7), pp.1881-1894. https://doi.org/10.1002/ijc.32980.

Persistent Link:

http://hdl.handle.net/11343/275623 